\title{
Design and Implementation of 3D Effect Web Page Based on JavaScript Technology
}

\author{
Huiyou Sun ${ }^{1}$, Shuangyuan Liª, Mingqian $\mathrm{Jia}^{1}$ \\ ${ }^{1}$ Jilin Institute of Chemical Technology, 45 Chengde Street, Longtan District, Jilin City, Jilin Province,China
}

\begin{abstract}
With the development of Internet technology, people's production and life are greatly influenced. Web design is widely used in Internet world. Web design and user experience are inseparable. In the pursuit of webpage layout and aesthetics, but also to add user interaction functions, Dynamic effects technology based on JavaScript can achieve user interaction requirements. The perfect combination of JavaScript dynamic effects and interactive features is the best way to achieve webpage effects. This paper expounds the application method of JavaScript, and studies the special effect application of JavaScript technology in webpages through examples.
\end{abstract}

Keywords: JavaScript; 3D effect; Web page technology

JavaScript is a literal, universal, prototype-based, objectoriented scripting language. The design principle is to provide code that can be inserted into different applications without taking up operating system and network resources. JavaScript has the following features and advantages: explanatory scripting language, simple development environment, note pad can be used to write code, good security and dynamic, cross-platform, and strong expansibility.

In this paper, the features of JavaScript technology are demonstrated and analyzed through examples. The application of JavaScript technology in the Web can design many personalized dynamic Web page effects. This paper expounds its design idea and provides the realization method of web 3D effects. The JavaScript effect technology is applied to web design, and its performance and design effect are analyzed in detail.

\section{Understand the Basics of JavaScript}

How JavaScript is used (direct mode and referential mode) $<$ script type = "application/javaScript" $>$ $<!$

function myFunction() \{ document.getElementById("myPar").innerHTML="Hell o World",

\}

var mychar = "This is a javaScript! Use direct insert! "

document.write (mychar+ " $<$ br/> ")

//-javaScript finish-->

$</$ script $>$

$<$ div id = "myPar" $></ d i v>$

The direct method is implemented by means of a pair of tags $<$ script $>$ direct mode $</$ script $>$ directly, and changes and adjusts the page by means of HTML writing rules and javaScript operation rules. When you need to make changes to the page, simply write the rules in the pair of labels. Of course, you can add a pair of tags, and writing rules in this tag is also an effective method ${ }^{[1]}$. (find the right pair of script tags for strict advice)

$<$ script type = "application/javaScript" $>$ this is the W3C's new standard for browser identification javaScript coding, which is interpreted by the browser by calling the corresponding interpreter ${ }^{[2]}$.

It is important to note: embedded script tags in the XHTML document (for example the PHP documentation), if in the absence of references to external files, should add the cdata statement in the script, cdata will tell the browser the document does not use XML parser for text data parsing, avoid is greater than and less than operators cause the browser to parse error ${ }^{[3]}$.

End of "//-JavaScript": the role of the double slash in the JavaScript language and <!---> is the same in the HTML language. It's called an annotation symbol. It's used to annotate and hide unrunning text. Document.write () function indicates to the corresponding information in parentheses in the output to a window, var is to declare a variable, sometimes not, the statement can also be used to code the rigorous and unnecessary trouble typically statement, because a lot of time in a javaScript document more than one or two variables, more conducive to the handling of each variable is clear statement. Note that $<$ script $><$ script $>$ this pair of tags is not fixed and can be included in anywhere of the labels $<$ head $></$ head $>$ or $<$ body $></$ body $>$. There are two common conventions. One is to add a pair of tags under the code of the plate. The other is to write the script tag directly at the beginning and end of the document body, regardless of the plate position ${ }^{[4]}$.

\footnotetext{
a Shuangyuan Li: 1sy@jlict.edu.cn
} 
function myFunction() \{

document.getElementById("myPar").innerHTML = "Hell o World",

\}

This is a custom javaScript function that tells the browser to execute the getElementById () function in the entire web document object model, locate the web element whose id value is myPar, and add the HTML element "Hello World!" to it. In the later research of 3D effects technology, this method of dynamically adding HTML to elements is applied.

$$
<\text { script } \operatorname{src}=\text { "url” type }=\text { "text/javaScript" }></ \text { script }>
$$

The example above is the basic format of javaScript reference way, through the same way of external links with CSS will all javaScript code written in a javaScript source file (general with js file extension), and by importing the language to import the entire source files to the index of javaScript on the page in the change, this approach can improve the utilization rate of the program code.

The URL represents the address that needs to be imported into the program file to find the program file through the address solution program. Again, such statements can be placed in the head or body of an HTML document, but in general we usually place such files in the first place. When the web page is opened, the first thing the web page does is to load the content, and such files can be put in the first place, which can be first loaded, and then start to display the content of the home page, so as to avoid the phenomenon of page crash when the web page loads ${ }^{[5]}$.

Assuming that you want to achieve the effect of the example in "direct insert mode", you can first create a JavaScript source file named "JavaScript. Js", which reads as follows:

Document write(" this is JavaScript! Use the direct insert method!');

You can call the program on a web page like this: $<$ scriptsrc $=$ "javaScript.js"type $=$ "text $/$ javaScript " $></$ script $>$, then you can achieve the same effect in the direct way.

You can also make the version of javaScript when importing files at the same time, such as: <script SRC $=$ "javaScript.js"type $=$ "text $/$ javaScript; Version $=4.18$ " $></ \mathrm{s}$ cript $>$, which tells the troubleshooter and maintainer that this is version $4.18,4$ is the basic version number, and .18 is the small version number.

Note: anything in the script tag that specifies the SRC attribute is ignored.

\section{Web 3D Implementation}

In the first part of this article, I introduced the basic application of JavaScript, and learned that JavaScript can realize simple user interaction and increase user experience. Here's an example of how JavaScript can be used in web effects.

Web 3D animation can attract the interest of visitors. What if the $3 \mathrm{D}$ effect is achieved through JavaScript technology? Detailed implementation methods are listed below.
$<$ !DOCTYPE html PUBLIC “-//W3C//DTD XHTML 1.0

Transitional//EN">

$<$ html $>$

$<$ head $>$

$<$ meta http-equiv="Content-Type" content="text $/$ html; charset $=$ utf- 8 " $>$

$<$ title $>$ Effect of $3 D$ navigation $</$ title $>$

$</$ head $>$

$<$ body $>$

$<$ style type $=$ "text/css">

body\{background:black\}

.point\{position:absolute;height:80px; \}

$</$ style $>$

$<$ script $>$

var $r=200, d v=0.01, w=100, x=400 ; y=100, p n=8 ; / / r \quad$ is radius, $d v$ is offset, $w$ is width of picture, $x$ is Menu abscissa, $y$ is menu ordinates, pn is number of menu pictures

var $\mathrm{pi}=$ Math.PI, $d=$ pi/2;

var $p d=$ Math.asin $(w / 2 / r), e d=p i * 2 / p n, s m ; / / p d$ is the inverse sine of each picture

function $\operatorname{goR}()\{$

var $o=$ document.getElementById('imground');

var arrimg $=$ o.getElementsByTagName('img');

for (var $n=0 ; n<$ arrimg.length; $n++)\{$

arrimg [n].onmouseout $=$ function() $\{$ sm $=$ setInterval('round Move()',50); // Move the mouse away from the menu arrimg $[n]$.onmouseover $=$ function() $\{$ clearInterval $(\mathrm{sm})\}$ // Mouse over the menu to stop the rotation

arrimg[n].onmousedown=function() $\left\{d v=d v^{*} 2\right\} / /$ Click on the menu to speed up the transition

\}

sm=setInterval('roundMove()',50);

\}

function roundMove()\{

var $v=$ document.getElementById('imground');

var arrimg $=$ v.getElementsByTagName('img');

var o,ta,strFilter;

for $($ var $n=1 ; n<=p n ; n++)\{$

$o=\operatorname{arrimg}[n-1]$;

ta $=$ Math.sin $\left(d+e d^{*} n\right) ; / /$ Gets the sine of the current offset strFilter $="$;

if $(\mathrm{ta}<0)\{/ /$ The sine value corresponds to negative radians, where the picture rotates to the back of the circle o.style.left $=$ Math.cos $\left(d+e d^{*} n-p d\right) * r+x+{ }^{\prime} p x^{\prime}$; // Passes in the abscissa of the current picture movement

\}

else \{// When the image rotates to the front of the circle o.style.left $=$ Math.cos $\left(d+e{ }^{*} n+p d\right) *_{r}+x+{ }^{\prime} p x^{\prime} ; / /$ Passes in the abscissa of the current picture movement

\}

o.style.top $=t a * 10+5+y+{ }^{\prime} p x^{\prime} ; \quad / /$ Passes in the vertical coordinate of the current image movement o.style.width=Math.abs (Math.cos $\left(d+e d^{*} n+p d\right)$ -

$\left.\operatorname{Math} \cdot \cos \left(d+e{ }^{*} n-p d\right)\right){ }^{*}+{ }^{\prime} p x$;

o.style.zIndex $=$ parseInt $($ ta $* 10)$;

if (o.style.zIndex<0) \{// Flip the image as it rotates to the back positionstrFilter $=$ 'FlipH(enabled:true)';

\}

else\{ // Displays the front of the image as it rotates to the front 


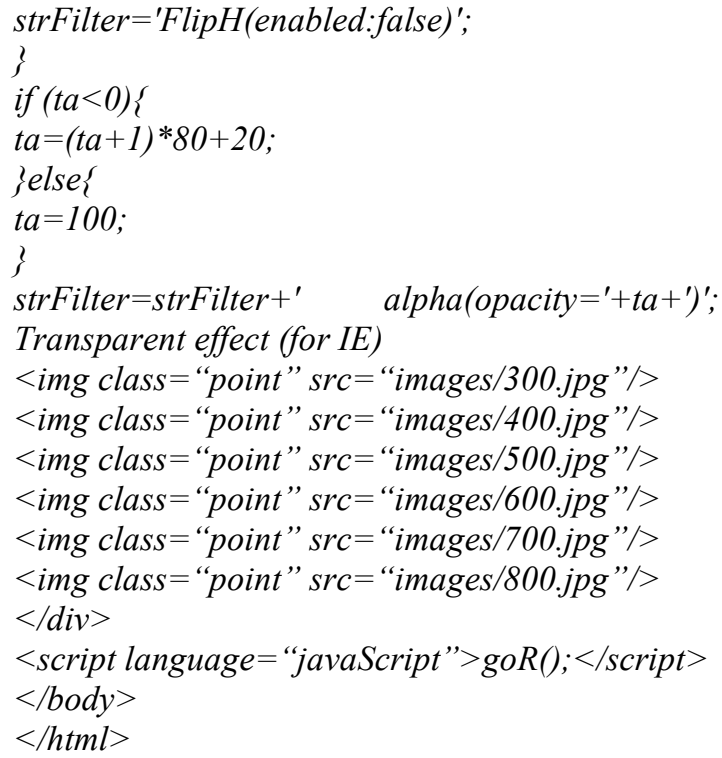

\section{Summarize}

JavaScript as a dynamic type and weak type of scripting language translation type, its browser based operations, support the distributed computing, and functions are performed on the client, do not need a specific environment for development, any JavaScript plain text editor can edit text, cross-platform, which can realize multiple platforms to share code, also reduce the network transmission, will be part of the information submitted data verification and validation on the client.

The use of special effects, as a demonstration method of JavaScript in the layout process, is to satisfy users' demands through dynamic display with the most intuitive o.style.opacity=ta/100; // Transparent effect (for non-IE) o.style.filter $=$ strFilter; // Add filter effects to menu images \} $d+=d v ; / /$ Increase offset

\}

$</$ script $>$

$<$ div id= "imground" $>$

$<$ img class = "point" src = "images/100.jpg"/>

$<$ img class $=$ "point" src="images/200.jpg"/>

visual impact through a series of editing and design techniques, such as picture composition and coordinate and point painting, under the condition of language features based on JavaScript.

\section{References}

1. Y. Cao, J. Wang, JavaScript Programming Analysis, Science \& Technology Association Forum (the semimonthly), 5, 62 (2009).

2. Y. Zhang, Exploration of Integrated Application of Javascript And Java Language in Dynamic Web Design, University Education, 8(1), 63-64 (2012).

3. J.S. Zheng, M. Jiang, Research on a Web Layout Method Based on DIV+CSS+JavaScript, Computer Knowledge and Skills, 17, 1556-1558 (2008).

4. S.W. Ping, Y.Y. Pan, X.J. Zhang, X.R. Du, Development of a Lightweight Animation Engine Based on HTML5 and JavaScript, Computer technology and development, 23(12), 5-10 (2013).

5. S.Y. Pang, Discussion on the Improvement of JavaScript Code Efficiency in Web Page Loading, China Academic Journal Electronic Publishing, Digital Technology \& Application, 07, 232 (2017). 